\title{
The Role of Human Epidermal Growth Factor Receptor (HER2/neu) in the Prognosis of Patients with Gastric Cancer
}

\author{
Mozaffar Aznab $^{1 *}$, Davood Maleksabet ${ }^{2}$, Sedigheh Khazaei ${ }^{3}$, Mansour Khazaei ${ }^{4}$, \\ Mansour Rezaei ${ }^{5}$
}

\begin{abstract}
Objective: Gastric cancer is one of the oncological challenges, and tendency toward target therapy in this cancer has been increased. Controversy still exists on prognostic value of HER2/neu expression and its relationship with clinicopathological characteristics and survival of gastric cancer patients. In this regard, the present study examined the status of HER2/neu in patients with gastric cancer and its prognostic effects. Methods: Pathological samples of 97 gastric cancer patients diagnosed over the last 8 or 9 years (from 2008 to the end of 2017) and treated with 5-fluorouracil, Docetaxel, and Cisplatin (TCF) were studied in this investigation. Patients were assigned to two groups according to their HER2/neu status. First group included patients with positive HER2/neu (Score 3) and second group involved patients with negative HER2/neu (Score 0 and 1). Patients were compared in terms of disease stage, survival rate, and mortality. Results: The mean age of patients was 58 years old. There were 75 men and 22 women in this study. In terms of disease stage, 4, 21, 41, and 31 patients were in stage I, II, III, and IV, respectively. Using IHC method, it was found that $27,23,25$, and 22 patients had HER2/neu expression with score 0 , score +1 , score $2+$ and score +3 , respectively. We discovered that expression of positive HER2/neu was associated with male sex. We also observed that survival and mortality rates following treatment initiation were significantly different between HER $2 /$ neu positive and negative gastric cancer patients $(\mathrm{P}<0.01)$. Conclusion: Evaluation of HER2/neu status in gastric cancer patients showed that HER2/neu 3+ expression could reduce the patients' survival. Therefore, it is recommended that patients who may benefit from trastuzumab, be treated. A clinical multi-center trial should be also considered for use of this drug in adjuvant cases.
\end{abstract}

Keywords: Gastric cancer- trastuzumab- HER2/neu3+ expression- Stage IV

Asian Pac J Cancer Prev, 20 (7), 1989-1994

\section{Introduction}

Gastric adenocarcinoma accounts for $90-95 \%$ of gastric malignancies, and has been considered as one of the most common causes of cancer-induced death throughout the world (Kamangar et al., 2006). About 2,800 new cases of cancer and 10,960 cases of cancer-induces death were reported in the United States in 2017 (American Cancer Society, 2017). Gastric adenocarcinoma is divided into spreading and intestinal subtypes (Guggenheim and Shah, 2011). The intestine type of gastric adenocarcinoma usually occurs in people over age 50 with higher prevalence among men. The incidence of its spreading type is more common at lower ages with higher prevalence among women (Guggenheim et al., 2004). The prevalence of its intestinal type has dramatically declined in the United States (Anderson et al., 2010). However, the incidence of the spreading type has not significantly changed over the past few decades.
The latter type has worse prognosis (Anderson et al., 2010). Based on 2005 statistics, most cases of gastric cancer have been observed in Japan, China, and Russia; while the least rates have been reported in Western developed countries (Crew and Neugut, 2006; Lindsey et al., 2012). The incidence of advanced gastric cancer in Japanese may be due to low rates of screening and early detection (Inoue and Tsugane, 2004). The prevalence of gastric cancer drastically differs in different populations, which can be attributed to the genetic differences and different lifestyles across various countries. Development of a cancerous cell is a multifactorial process affected by both environmental and genetic factors (Lichtenstein et al., 2000). Helicobacter pylori infection, lifestyle and nutrition, can be mentioned as the environmental risk factors for gastric cancer (Tsugane and Sasazuki, 2007). The intestinal type of gastric cancer is often associated with Helicobacter pylori infection (Atherton, 2006; Kusters et al., 2006). Long-term consumption of high 
concentrations of nitrates in dried, smoked, or salted food also has been considered as one of the risk factor for the intestinal type of gastric cancer. Several factors can facilitate the growth of nitrate-converting bacteria in the stomach, namely decreased gastric acidity as well as history of stomach surgery (antrectomy), atrophic gastritis, and pernicious anemia. Gastric cancer is developed in sporadic or inherited types following the presence of hereditary syndromes such as FAP, HDGC, and Lynch. Gastric cancer with HDGC origin is a predominant autosomal disease in a way that about $30 \%$ of patients with this disease have a mutation in one of the E-Cadherin or CDH1 tumor suppressor genes (Brooks-Wilson et al., 2004; Benusiglio et al., 2013). The most common symptoms appearing at the disease onset are weight loss and continuous abdominal pain. Dysphagia is common in patients with proximal gastric cancer or those having cancer at the site of gastro-esophageal junction. Sufferers may also experience early feelings of fullness and nausea. Advanced distal gastric cancer is manifested by obstructive symptoms. Occult blood with or without iron deficiency anemia, hematemesis, and melena are among the other symptoms of gastric cancer. Treatment of gastric adenocarcinoma in its early stages is done through surgery. Multidrug chemotherapy before (Chua and Cunningham, 2007) and after surgery, as well as post-surgery chemotherapy and radiotherapy (Smalley et al., 2012) or adjuvant chemotherapy (World GI Press Release, 2016; Aznab et al., 2017) are among the other treatments indicated to reduce the recurrent rate and enhance the survival rate of these patients. Since gastric adenocarcinoma is mostly diagnosed in its advanced stages, and there is a risk of recurrence due to micro metastasis even in localized cases, treatments such as surgery and chemotherapy usually fail to significantly increase the survival of these patients. As the important role of targeted therapy in other cancers (whether in adjuvant or in the metastatic states) for increasing the survival of patients, it is better to pay more attention to this issue in gastric cancer. Positive (HER2/neu) can be regarded as a promising therapeutic goal for new drug combinations, such as trastuzumab, since its efficacy in breast cancer has been proven. The role of trastuzumab in the treatment of positive HER2/neu gastric cancer patients in a metastatic condition is under investigation and various results have been reported. Application of this drug along with chemotherapy drugs simultaneously has not been evaluated in a localized stage (adjuvant). It is supposed that it may be effective for preventing the disease recurrence and increasing the survival of gastric cancer patients. Therefore, this study aimed to investigate the relationship between expression of HER2/neu and survival rate of the gastric cancer patients.

\section{Materials and Methods}

In this investigation, pathological samples of 97 gastric cancer patients diagnosed over the last 8 or 9 years (from 2008 to the end of 2017) and treated with 5-fluorouracil, Docetaxel, and Cisplatin (TCF) were studied. Patients' pathological samples were collected from laboratories of different hospitals in Kermanshah, Kurdistan, Ilam, Lorestan, and southern parts of Hamedan provinces. Then, 4-micron cuts were prepared from paraffin blocks of these samples for $\mathrm{H}$ and $\mathrm{E}$ staining; then the cuts were reused for immunohistochemistry (IHC) staining of HER2/neu.

\section{Paraffin section}

The antibody can be used for labeling of paraffin-embedded tissue section fixed in formalin. Optimal results are obtained with EnVision TM FLEX Target Retrieval Solution Low pH, Code K8005 or Dako Target Retrieval Solution, Low pH, Code S1699/S1700. Alternatively, EnVision TM FLEX Target Retrieval Solution, High pH, Code K8000/K8010 may be used at a different antibody dilutions.

\section{Dilution}

Polyclonal Rabbit Anti-Human c-erbB-2 Onco-protein, Code A0485, may be used at a dilution range of 1:600-1:800 or $1: 1000-1: 1200$.

After observing the slides by the pathologist, the positive or negative HER2/neu results were recorded in data collection forms. Other patients' information such as age, sex, stage of the disease at the time of diagnosis, the patient's lifetime after diagnosis, the onset of treatment, and patient's final condition (being alive or dead) was also recoded. Patients were compared in terms of the stage of the disease and survival rate based on the HER2/neu scores $(0,+1+, 2+$ and $3+)$. The scoring method for gastric cancer is shown in Table 1. Data processing and analysis were conducted using SPSS (version 21). Chi-square test was run to compare nominal variables while independent t-test was employed for quantitative variables. If the data were not normal according to KS normality test, u-MannWhitney non-parametric test was applied. The significance level was considered as 0.05

\section{Results}

The patients' age ranged from 27 to 73 years old, and the mean age of the patients was 58 years old. $70 \%$ of the patients had zero and one ECOG status. In terms of sex, $75(77 \%)$ patients were males and $22(23 \%)$ were females. Based on the TNM system, patients' stage of the disease at the time of diagnosis was as follows: 2 patients $(2 \%)$ were in IA stage, $2(2 \%)$ were in IB stage, $12(12.37 \%)$ were in IIA stage, $9(9.2 \%)$ were in IIB stage, $11(11.34 \%)$ were in IIIA stage, $11(11.34 \%)$ were in IIIB stage, 19 $(19.58 \%)$ were in IIIC stage, and 31 (31.9\%) were in IV stage. HER2/neu expression was evaluated by IHC and the following results were obtained: score 0 (negative) was observed in 27 patients; score $1+$ (negative) was detected in 23 patients; score $2+$ was observed in 25 patients; and score $3+$ was found in 22 patients. Statistically, a significant difference was found between patients in stage IV and those in stage II and III concerning the survival rate $(\mathrm{P}<0.000)$. With respect to overall survival, a significant difference was also observed between patients in stages II and III $(\mathrm{P}<0.03)$. The initial site of the tumor was gastric cardia, lesser curvature, greater curvature pyloric anturum, cardia, lesser curvature, and pan-gastric in 27,12 , 
Table 1. Her 2 nu Scoring in Gastric Cancer

\begin{tabular}{lll}
\hline 0 & negative no reactivity or no membranous reactivity in any tumor cell & Negative \\
$1+$ & $\begin{array}{l}\text { Tumor cell cluster with a faint/barely perceptible membranous reactivity irrespective of percentage of tumor } \\
\text { cells stained }\end{array}$ & Negative \\
$2+$ & $\begin{array}{l}\text { Tumor cell cluster with a weak to moderate complete, basolateral or lateral membranous reactivity irrespective } \\
\text { of percentage of tumor cells stained }\end{array}$ & Equivocal \\
$3+$ & $\begin{array}{l}\text { cell cluster with a strong complete, basolateral or lateral membranous reactivity irrespective of percentage of } \\
\text { tumor cells stained }\end{array}$ & Positive \\
\hline
\end{tabular}

3, 42, 2, and 11 patients, respectively. The most prevalent location of the tumor was the distal area. Patients' clinical and demographic characteristics are shown in Table 2.A statistically significant difference was observed between the patients whose tumors were in cardia and those in whom tumors were located in pyloric antrum patients in term of overall survival, in a way that it was higher in the latter group $(\mathrm{P}<0.01)$ (Figure 1). Moreover, a significant difference was detected in overall survival of patients whose lesion site was pan-gastric and that of patients whose lesion site was in pyloric antrum $(\mathrm{P}<0.04)$ (Figure $1)$. The difference in overall survival was significant after treatment initiation between gastric cancer patients with positive HER2/neu (score 3) and negative HER2/ neu (score 0 and 1$)(p<0.013)$. In terms of disease stage

Table 2. Characteristics of Patients with Gastric Cancer

\begin{tabular}{|c|c|c|}
\hline Variables & Total Population & Percent $\%$ \\
\hline Means age (year) & 58 year & \\
\hline \multicolumn{3}{|l|}{ Sex } \\
\hline Male (n) & 75 & 77.00 \\
\hline Female (n) & 22 & 23.00 \\
\hline Stage & Number & \\
\hline Stage IA & 2 & 2.00 \\
\hline Stage IB & 2 & 2.00 \\
\hline Stage IIA & 12 & 12.37 \\
\hline Stage IIB & 9 & 9.20 \\
\hline Stage III A & 11 & 11.34 \\
\hline Stage III B & 11 & 11.34 \\
\hline Stage III C & 19 & 19.58 \\
\hline Stage IV & 31 & 31.90 \\
\hline \multicolumn{3}{|l|}{ Tumor location } \\
\hline gastric cardia & 27 & 27.80 \\
\hline lesser curvature & 12 & 12.30 \\
\hline greater curvature & 3 & 3.00 \\
\hline pyloric anturum & 42 & 43.20 \\
\hline cardia and lesser curvature & 2 & 2.00 \\
\hline pan-gastric & 11 & 11.30 \\
\hline \multicolumn{3}{|l|}{ HER2/ neu scoring } \\
\hline 0 & 27 & 27.80 \\
\hline $1+$ & 23 & 23.70 \\
\hline $2+$ & 25 & 25.70 \\
\hline $3+$ & 22 & 22.60 \\
\hline \multicolumn{3}{|l|}{ ECOG status } \\
\hline $0-1$ & 70 & 70.00 \\
\hline$>2$ & 27 & 30.00 \\
\hline
\end{tabular}

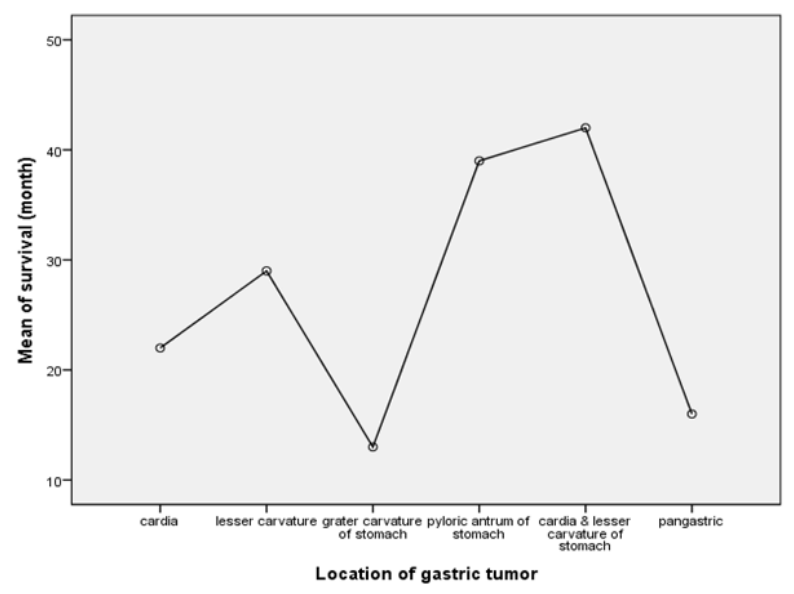

Figure 1. Survival of Patients with Gastric Cancer in Terms of Location of Tumor

at the time of diagnosis, significant relationship was observed between positive HER2/neu and negative HER2/ neu patients $(\mathrm{P}<0.06)$. The overall survival of patients possessing score 0,1 , and 2 was significantly different with that of patients at stage $3(\mathrm{P}<0.03)$ (Figure 2). Finally, 47 patients survived $(48.5 \%)$ and 50 of them $(51.5 \%)$ died; however, two of these deaths were due to non-cancer reasons. Overall survival of our patients in terms of disease stage is shown in Figure 3.

\section{Discussion}

The significance of HER2 gene in gastric cancer was first reported in 1986 (Sakai et al., 1986). Numerous studies have confirmed the prevalence of HER2 in gastric

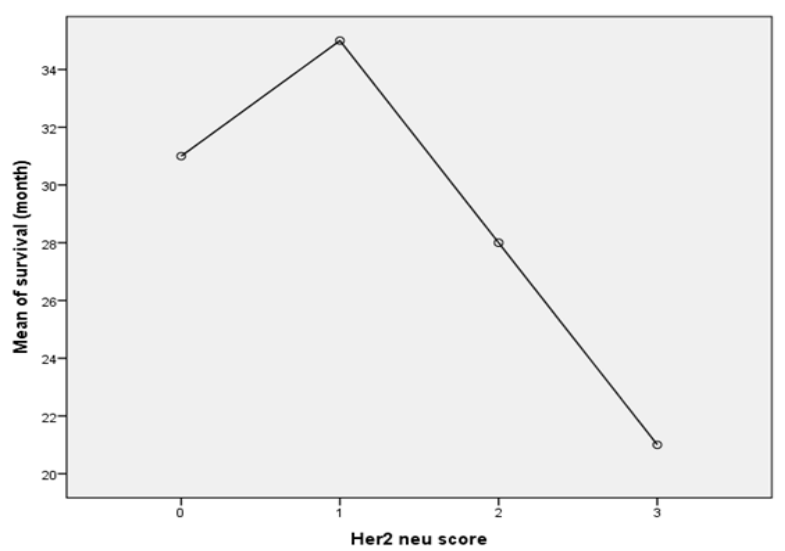

Figure 2. Survival of Patients with Gastric Cancer in Terms of HER2/neu Score. Negative=0; Negative $=1+$ Equivocal $=2+$; Positive $=3+$ 


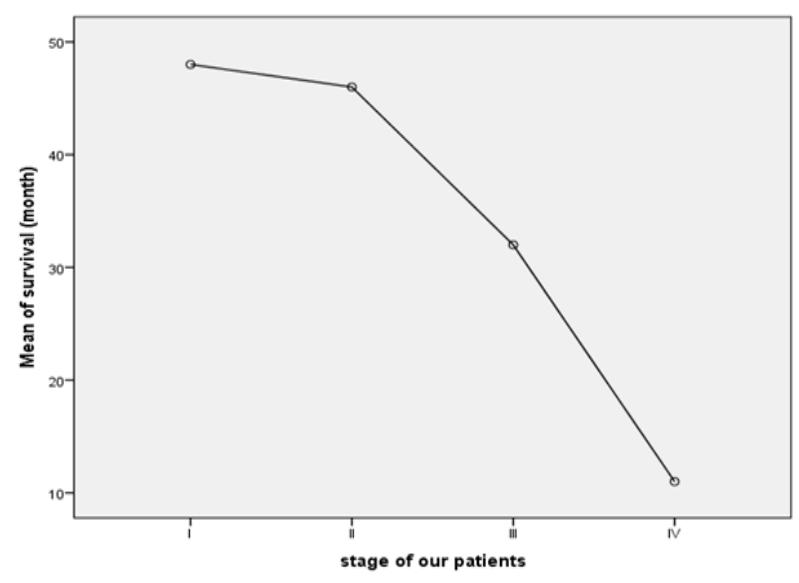

Figure 3. Overall Survival of Patients in Terms of Disease stage

cancer patients throughout the world. For instance, Jørgensen and Hersom, (2012) revealed that the rate of positive HER2neu was $4-53 \%$ based on just IHC, but when FISH was also considered, this rate changed to $9-18 \%$. The rate of positive HER2 was obtained as $23 \%$ in our study based on IHC. Shi-Yan et al., (2011) studied 145 tissue samples of gastric adenocarcinoma and concluded that positive HER2/neu was correlated with depth of invasion, high stage of the disease (based on TNM), involvement of lymph nodes, and far metastasis. They also indicated that the status of HER2/neu gene expression had no significant relationship with age, sex, or tumor site. Based on our finding, a significant relationship existed in positive expression of HER2/neu gene between men and women (46.2\% in men vs. none in women) $(\mathrm{P}<0.05)$. Therefore, it can be concluded that positive HER2 expression is correlated by male gender, which is in agreement with findings of other studied. Furthermore, we found a significant difference among different stages of the disease with respect to positive HER2 expression $(\mathrm{P}<0.05)$, in a way that more negative HER expression was observed in lower stages of the disease. Hence, we detected a significant relationship between positive HER2 expression and gastric adenocarcinoma stage, which is found in many other studies in this regard (Yan et al, 2010; Vui Heng et al., 2016). Begnami et al., (2011) investigated 211 tissue samples of gastric adenocarcinoma patients by IHC method in search for HER2. They realized a significant association between HER 2 and tumor invasion depth, involvement of lymph node, and high stage of the tumor. Moreover, HER2 was significantly associated with bas prognosis of the patients according to findings of aforementioned study.

Park et al., (2006) studied 182 treated gastric cancer patients in 2006. They concluded that the means of survival duration were 922 and 3243 days in positive and negative HER2/neu groups, respectively. Moreover, the rates of survival for 3 years were $42.9 \%$ and $74 \%$ in positive and negative HER2/neu groups, respectively. The relationship between positive HER2 marker and gastric adenocarcinoma was also confirmed in 2003 by Garcia et al., (2003). Nevertheless, these results were not verified in some other studies. For instance, Sheng et al., (2013) investigated tissue samples of gastric adenocarcinoma in 726 Chinese patients by IHC and FISH methods and showed no significant difference in 3-year survival of HER2/neu positive and negative groups. In 2012, Janjigian et al., (2012) examined 381 tissue samples of gastric adenocarcinoma by IHC and FISH methods and found 78 positive samples $(20 \%)$ in terms of HER2/neu expression. The overall survival of gastric cancer patients possessing positive HER2/neu was higher than that of negative HER2/neu; however, this difference was not significant according to aforementioned study. They also observed that HER2/neu state could not independently determine the prognosis based on multivariable analysis. Based on our results, the difference in survival rates of positive and negative HER2/neu expressions were significant after treatment $(\mathrm{P}<0.013)$, which is in line with findings of some of other researcher. In a nutshell, it seems that HER2/neu status can be related with stage of gastric cancer. Hence, conducting HER2/neu investigation in all patients with gastric cancer is recommended during pathological evaluations.

Numerous studies recommended the administration of trastuzumab in gastric cancer patients with positive HER2/neu, and many studies have suggested the addition of trastuzumab to chemotherapy drugs. Cunningham and Okines, (2010) found that addition of Herceptin (trastuzumab) to chemotherapy regimen of gastric cancer with positive HER2/neu would result in significant improvement in response to treatment and mean of patients' survival. However, the best study in this regard was that of Trastuzumab for Gastric Cancer (TOGA). In TOGA trial (Bang et al., 2010), it was shown that addition of trastuzumab to chemotherapy regimen of cisplatin and flourouacil in metastatic gastric cancer patients, in comparison with chemotherapy alone, led to enhancement of response to disease, life time, and recurrence-free survival. In this trial, those with HER2/ neu score 3+ or FISH+ experienced increased life time of 4.2 months (from 11.8 month for chemotherapy alone to 16 month with addition of trastuzumab to chemotherapy regimen). Therefore, the adminstration of trastuzumab along with Capecitabine or flourouaciland cisplatin in Europe and some other areas has been done in metastatic cases of gastric or cardia adenocarcinoma. In 2010, Tetsuji, (2010)investigated 24 patients and found that addition of trastuzumab to chemotherapy regimen of advanced gastric adenocarcinoma patients with positive HER2/neu increased their lifetime significantly. The mean of overall survival of these patients increased from 11.8 months to 16 months in their study. Gomez- Martin et al., (2013) addressed the rate of HER2/neu expression and its relationship with trastuzumab plus chemotherapy treatment results among gastric cancer patients in 2013. They showed that the higher the expression of HER2/ neu, the higher the sensitivity to treatment and overall survival of gastric cancer treated with trastuzumab plus chemotherapy. The recurrence of the disease in stages I, II, and III is the other challenge facing the sufferers. The use of target therapies can reduce the rate of recurrence due to micro-metastasis theory. Hence, it is recommended to conduct a multi-center large trial to study the effect 
of administration of trastuzumab in localized stages on reduction of the recurrence and mortality of this disease.

In conclusion evaluation of HER2/neu status in gastric cancer patients showed that expression of HER2/ neu +3 resulted in reduction of patients' overall survival. We also found a significant relationship between HER2/ neu status and stage of gastric cancer; therefore, it is recommended that patients, who may benefit from this treatment, receive it. Furthermore, investigation of the efficacy of trastuzumab administration in localized stages in deceasing the recurrence and mortality of this disease is suggested.

\section{Acknowledgements}

The authors would like to thank all staff working in Oncology Hematology department of Imam Reza Hospital.

\section{Conflict of interest}

The authors declared no potential conflicts of interest.

\section{Funding/Support}

No financial support.

\section{References}

American Cancer Society: Cancer Facts and Figures 2017. Atlanta, Ga: American Cancer Society, 2017.

Anderson WF, Camargo MC, Fraumeni JF Jr, et al (2010). Age-specific trends in incidence of noncardia gastric cancer in US adults. JAMA, 303, 1723-8.

Atherton JC (2006). The pathogenesis of Helicobacter pyloriinduced gastro-duodenal diseases. Annu Rev Pathol, 1, 63-96

Aznab M, Beiki O, Pia KE, et al (2017). Evaluation the survival of patients with gastric cancer treated with adjuvant or palliative chemotherapy. J Gastrointest Cancer, 48, 31-7.

Bang Y, Van Cutsem E, Feyereislova A, et al (2010).Trastuzumab in combination with chemotherapy versus chemotherapy alone for treatment of HER2-positive advanced gastric or gastro-oesophageal junction cancer (ToGA): a phase 3, open-label, randomised controlled trial. Lancet, 376, 687-97.

Benusiglio PR, Malka D, Rouleau E, et al (2013). CDH1 germline mutations and the hereditary diffuse gastric and lobular breast cancer syndrome: a multicentre study. $\mathrm{J} \mathrm{Med}$ Genet, 50, 486-9.

Begnami MD, Fukuda E, Fregnani JH, et al (2011). Prognostic implications of altered human epidermal growth factor receptors (HERs) in gastric carcinomas: HER2 and HER3 are predictors of poor outcome. J Clin Oncol, 29, 3030-6.

Brooks-Wilson AR, Kaurah P, Suriano G, et al (2004). Germline E-cadherin mutations in hereditary diffuse gastric cancer: Assessment of 42 new families and review of genetic screening criteria. J Med Genet, 41, 508-17.

Chua YJ, Cunningham D (2007). The UK NCRI MAGIC trial of perioperative chemotherapy in resectable gastric cancer: implications for clinical practice. Ann Surg Oncol, 14, 2687-90.

Crew KD, Neugut AI (2006). Epidemiology of gastric cancer. World J Gastroenterol, 12, 354-62.

Cunningham D, Okines AFC (2010). Trastuzumab in gastric cancer. Eur J Cancer, 46, 1949-59.

Gomez-Martin C, Plaza JC, Pazo-Cid R, et al (2013). Level of HER2 gene amplification predicts response and overall survival in HER2-positive advanced gastric cancer treated with trastuzumab. J Clin Oncol, 31, 4445-52.

Guggenheim DE, Shah MA(2013). Gastric cancer epidemiology and risk factors. $J$ Surg Oncol, 107, 230-6.

Garcia I, Vizoso F, Martin A, et al (2003). Clinical significance of the epidermal growth factor receptor and HER2 receptor in resectable gastric cancer. Ann Surg Oncol, 10, 234-41.

Guggenheim DE, Shah MA, Christopher D, et al (2004). Differential trends in the intestinal and diffuse types of gastric carcinoma in the United States, increase in the signet ring cell type. Arch Pathol Lab Med, 128, 1973-2000.

Inoue M, Tsugane S (2005). Epidemiology of gastric cancer in Japan. Postgrad Med J, 81, 419-24.

Janjigian YY, Werner D, Pauligk C, et al (2012). Prognosis of metastatic gastric and gastroesophageal junction cancer by HER 2 status: a European and USA International collaborative analysis. Ann Oncol, 23, 2656-62.

Jørgensen JT, Hersom M (2012). HER2 as a prognostic marker in gastric cancer - A systematic analysis of data from the literature. J Cancer, 3, 137-44

Kamangar F, Dores GM, Anderson WF (2006). Patterns of cancer incidence, mortality, and prevalence across five continents: defining priorities to reduce cancer disparities in different geographic regions of the world. J Clin Oncol, 24, 2137-50.

Katherine DC, Alfred IN (2006). Epidemiology of gastric cancer. World J Gastroenterol, 12, 354-62.

Kusters JG, van Vliet AH, Kuipers EJ (2006). Pathogenesis of Helicobacter pylori infection. Clin Microbiol Rev, 19, 449-90.

Lindsey AT, Freddie B, Rebecca LS, et al (2012). Global cancer statistics. CA Cancer J Clin, 65, 87-108.

Lichtenstein P, Holm NV, Verkasalo PK, et al (2000). Environmental and heritable factors in the causation of cancer-analyses of cohorts of twins from Sweden, Denmark, and Finland. N Engl J Med, 13, 78-85.

Lindsey AT, Freddie B, Rebecca LS, et al (2012). Global cancer statistics. CA Cancer J Clin, 65, 87-108.

Park DI, Yun JW, Park JH, et al (2006) HER-2/neu amplification is an independent prognostic factor in gastric cancer. Dig Dis Sci, 51, 1371-9.

Sakai K, Mori S, Kawamoto T, et al (1986). Expression of epidermal growth factor receptors on normal human gastric epithelia and gastric carcinomas. J Natl Cancer Inst, 77, 1047-52.

Shah MA, Khanin R, Tang L, et al (2011). Molecular classification of gastric cancer: a new paradigm. Clin Cancer Res, 17, 2693-2701.

Sheng D WQ, Huang JM, Ying N, et al (2013). HER2 status in gastric cancers: a retrospective analysis from four Chinese representative clinical centers and assessment of its prognostic significance. Ann Oncol, 24, 2360-4.

Shi-Yan Y, Ying H, Jian-Gao F, et al (2011). Clinicopathologic significance of HER-2/neu protein expression and gene amplification in gastric carcinoma. World $J$ Gastroenterol, 17, 1501-6.

Smalley SR, Benedetti JK, Haller DG, et al (2012). Updated analysis of SWOG-directed intergroup study 0116: a phase III trial of adjuvant radiochemotherapy versus observation after curative gastric cancer resection. J Clin Oncol, 30, 2327-33.

Tetsuji F (2010). Trastuzumab for gastric cancer treatment. Lancet, 376, 1735.

Tsugane S, Sasazuki S (2007). Diet and the risk of gastric cancer: review of epidemiological evidence. Gastric Cancer, $\mathbf{1 0}$, 75-83.

Vui Heng C, Pemasari U, Telisinghe F, et al (2016). Profile of 
Mozaffar Aznab et al

HER2 + ve gastric cancers in Brunei Darussalam. Asian Pac $J$ Cancer Prev, 17, 2555.

World GI (2016) Press Release: Chemoradiotherapy after surgeryfor gastric cancer shows similar outcomes to post-operative chemotherapy. 30 Jun 2016.

Yan B, Yau EX, Bte Omar SS, et al (2010). A study of HER2 gene amplification and protein expression in gastric cancer. $J$ Clin Pathol, 63, 839-42.

\section{(ब) $(\mathbb{\theta}$}

This work is licensed under a Creative Commons AttributionNon Commercial 4.0 International License. 